\title{
Inhibition of MMP-9 using a pyrrole-imidazole polyamide reduces cell invasion in renal cell carcinoma
}

\author{
AYA SATO $^{1}$, HIROKI NAGASE ${ }^{2}$, DAISUKE OBINATA ${ }^{1}$, KYOKO FUJIWARA $^{3}$, NOBORU FUKUDA ${ }^{3}$, \\ MASAYOSHI SOMA $^{3}$, KENYA YAMAGUCHI ${ }^{1}$, NOZOMU KAWATA ${ }^{1}$ and SATORU TAKAHASHI ${ }^{1}$ \\ ${ }^{1}$ Department of Urology, Nihon University School of Medicine, Itabashi-ku, Tokyo 173-8610; \\ ${ }^{2}$ Department of Cancer Genetics, Chiba Cancer Center Research Institute, Chuo-Ku, Chiba 260-8717; \\ ${ }^{3}$ Department of General Medicine, Nihon University School of Medicine, Itabashi-ku, Tokyo 173-8610, Japan
}

Received April 9, 2013; Accepted July 25, 2013

DOI: $10.3892 /$ ijo.2013.2073

\begin{abstract}
We investigated the clinical significance of the expression levels of matrix metalloproteinase 9 (MMP-9) in renal cell carcinoma ( $\mathrm{RCC}$ ). In addition, we validated the efficacy of pyrrole imidazole polyamide (PIP) targeting MMP-9 on inhibiting proliferation and invasion of RCC. We evaluated the expression levels of MMP-9 in 249 RCC specimens by immunostaining and analyzed the association between MMP-9 expression levels and cancer-specific survival. Furthermore, in a human RCC cell line, Caki-2, we tested the effect of a couple of PIPs targeting MMP-9 one recognizing an $N F-\kappa B$ binding site (MMP-9-NF- $\kappa$ B PIP) and another for the AP-1 binding site (MMP-9-AP-1 PIP) in the MMP-9 promoter. The expression levels of MMP-9, proliferative activity and invasive capability were tested by quantitative PCR, WST8 assay and Matrigel invasion assay, respectively. By immunostaining of the clinical specimens, strong MMP-9 staining was proven to be a significant predictor of poor prognosis for cancer-specific survival $(\mathrm{P}<0.01)$. In Caki-2 cells, MMP-9-NF- $\kappa$ B PIP significantly reduced the expression levels of MMP-9 mRNA and inhibited cell invasion, but did not affect the cell proliferation activity. On the other hand, no effect was found in MMP-9-AP-1 PIP on MMP-9 mRNA expression, cell proliferation and invasion. We confirmed the inhibitory effects of MMP-9-NF- $\mathrm{B}$ PIP on the expression of MMP-9 and subsequent invasion of Caki-2 cells. Since it was clearly shown that high MMP-9 expression levels were associated with poor prognosis of RCC, MMP-9 is a potential candidate target for RCC treatment. Transcription therapy using a minor groove binder, such as NF- $\kappa \mathrm{B}$ PIP, may be a potential therapeutic agent for RCC, although further investigation is required.
\end{abstract}

Correspondence to: Dr Hiroki Nagase, Department of Cancer Genetics, Chiba Cancer Center Research Institute, 666-2 Nitona-Cho, Chuo-Ku, Chiba 260-8717, Japan

E-mail: hnagase@chiba-cc.jp

Key words: pyrrole-imidazole polyamide, matrix metalloproteinase, renal cell carcinoma

\section{Introduction}

New agents including the small molecule targeted inhibitors like sunitinib, sorafenib and temsirolimus, for advanced renal cell carcinoma (RCC) have been developed based on a biological process in the RCC carcinogenesis. However, they are often accompanied by adverse effects that occasionally require dose reduction or discontinuation. Tenacious cancer cells subsequently become resistant to the same drug treatment. Therefore, development of new molecular targeting agents as novel targets with minimal adverse effects is still needed (1-3).

The permeation to basement membrane and the extracellular matrix of neoplastic cells is regarded as the most important step in metastatic process. MMP is a group of enzymes that degenerate various elements of extracellular matrix including elastin, gelatin, agrican, fibronectin and laminin (4,5). Furthermore, an impact of MMP on the early proliferation of tumor cells of metastatic focus has been reported. In addition, tissue inhibitor of MMP (TIMP), which is an endogenous MMP inhibitor participates in the activity of MMP and disproportion between TIMP and MMP modulates the progression of tumors $(6,7)$.

Among these MMPs, MMP-9 degenerates type IV collagen, gelatinized types I and II collagen and participates in the permeation of basement membrane by tumor cells. The expression of MMP-9 is increased in malignant tumors in comparison with benign or non-invasive tumors $(7,8)$. In addition, overexpression of MMP-9 is confirmed in renal cell carcinoma (8-12). Kawada et al reported association of MMP-9 expression and poor prognosis using clinical specimen of renal cell carcinoma $(13,14)$. Therefore, we expected the new therapeutic drug that inhibits activity of MMP-9 may have an antitumor effect for renal cell carcinoma.

Pyrrole-imidazole polyamides (PIP) are powerful gene regulating compounds, which can bind to the minor groove of DNA double strand in a sequence-specific manner. They are composed of two aromatic amino acids [N-methylpyrrole (Py) and N-methylimidazole (Im)]. The combination of Im/ Py recognizes GC in the DNA double-helix, Py/Py recognizes TA and AT and arrangements of these combinations made it possible to bind to a variety of sequence. Since they can bind DNA with higher affinity and specificity than the usual DNA binding proteins, PIPs designed to recognize the binding 
Table I. Patient background ( $\mathrm{n}=249$, age range 24-85. with an average of 60 years).

\begin{tabular}{lrr}
\hline Category & $\mathrm{N}$ & $\%$ \\
\hline Gender & 185 & \\
Male & 64 & 74 \\
Female & & 26 \\
Cell type & 203 & \\
Clear & 46 & 82 \\
Non-clear & & 18 \\
Fuhrman's grade & 90 & \\
1 & 108 & 36 \\
2 & 51 & 21 \\
$3+4$ & & \\
Stage & 135 & 54 \\
1 & 39 & 16 \\
2 & 41 & 16 \\
3 & 34 & 14 \\
4 & &
\end{tabular}

domain of transcription factors could inhibit the expression of downstream genes. Precise binding to a known sequence may make it possible to reduce adverse events, therefore PIP is expected to become a new molecular targeting agent (15-18). Previously, we reported that PIP targeting for MMP-9 inhibit the migration and invasion of colon cancer cells in vitro and also inhibits their metastasis in vivo (19).

In the present study, we examined MMP-9 immunoreactivity in clinical specimens and analyzed the degree of association and matched cancer-specific survival. Furthermore, we investigated effects of PIP on the inhibition of MMP-9 expression and resulting inhibition of cellular invasion by using human renal cell carcinoma cell line Caki-2.

\section{Materials and methods}

Tissue samples. Two hundred and forty-nine surgical samples with informed consent were obtained at Surugadai Nihon University Hospital from 1990 to 2003 (Table I). All of the studies using these specimens were performed under the approval of Nihon University School of Medicine Ethics Review Board (IRB no. 106-1).

Immunohistochemistry. Immunostaining for MMP-9 were performed and the degree of immunoreactivity was analyzed to find the association between cancer-specific survival by using Kaplan-Meier method with log-rank test. For immunostaining, formalin-fixed, paraffin-embedded 4- $\mu \mathrm{m}$ thick samples placed on silane coat slide glass (Dako, Carpinteria, CA, USA) were used. After, retrieval with $500 \mathrm{~W}$ microwave, slides were processed with $3 \%$ hydrogen peroxide and $5 \%$ non-fat milk phosphate buffered saline (PBS) to block non-specific antibodies. Rabbit polyclonal matrix metalloproteinase-9 antibody (Cell Signaling Technology, USA) was used as a primary antibody. Afterwards, samples were visualized by simple stain MAX-PO (Nichirei, Tokyo, Japan). The staining intensity of MMP-9 was stratified using a 4-grade scale, with grade 1 indicating the absence of immunostaining or faint membranous staining of rare tumor cells, grade 2 indicating membranous staining in most tumor cells, grade 3 indicating diffuse membranous and/or cytoplasmic staining in groups of tumor cells and grade 4 indicating significant cytoplasmic staining in most tumor cells (Fig. 1). For the evaluation of immunohistochemical staining, intensities of grades 1 and 2 were considered weak expressions of each protein, whereas grades 3 and 4 were considered strong.

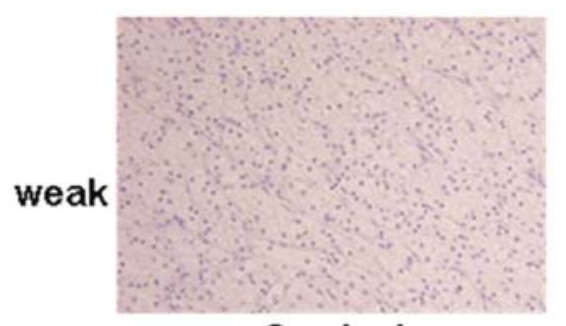

Grade 1

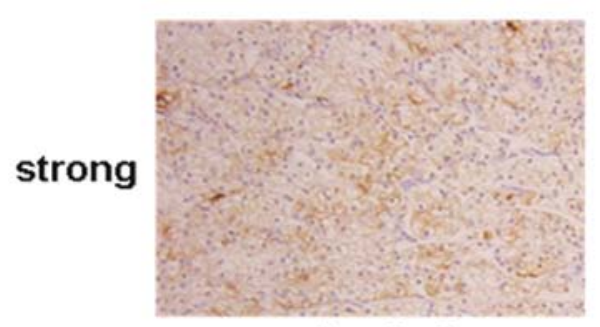

Grade 3

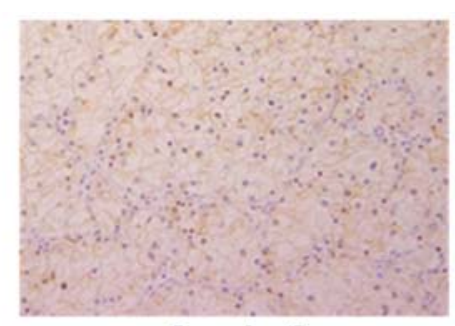

Grade 2

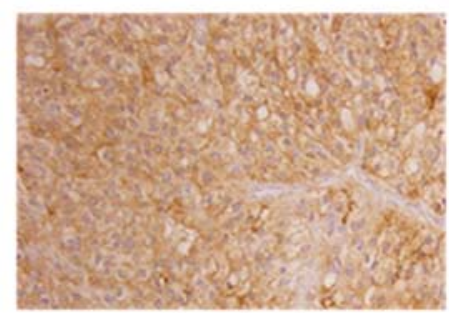

Grade 4

Figure 1. Immunohistochemical detection of MMP-9 in RCC specimens. MMP-9 protein expression in RCC tissues was detected immunohistochemically and the degree of MMP-9 immunoreactivity was classified as weak (grades 1 and 2) and strong (grades 3 and 4). Grade 1, absence of staining or faint membranous staining of rare tumor cells; grade 2, membranous staining in most tumor cells; grade 3, diffuse membranous and/or cytoplasmic staining in groups of tumor cells; grade 4, significant cytoplasmic staining in most tumor cells. Representative images are shown (original magnification, $\mathrm{x} 200$ ). 


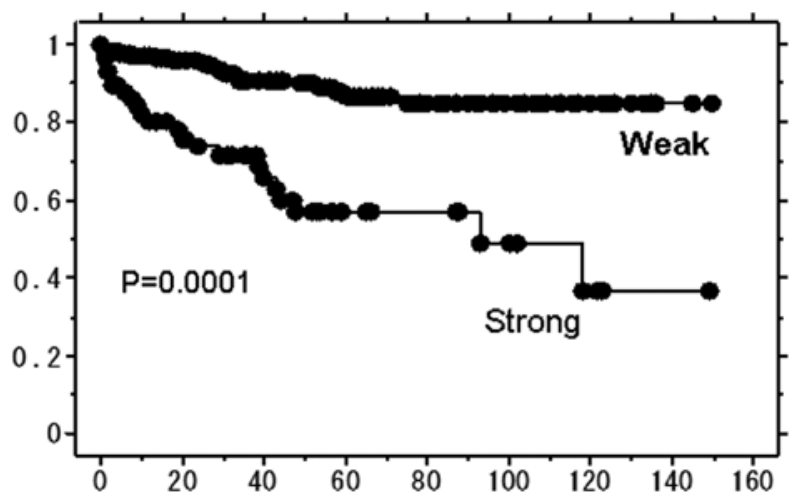

Figure 2. Kaplan-Meier curve of the RCC patients with weak and strong immunoreactivity for MMP-9. Survival curves were drawn using Kaplan-Meier method for 194 of weak and 55 of strong MMP-9 staining groups. Significant difference of cancer-specific survival rate $(\mathrm{P}=0.0001)$ was observed between weak and strong immunoreactivity for MMP-9.

Synthesis of PIP targeting human MMP-9. The expression of MMP-9 gene is known to be regulated by the transcription factors NF- $\mathrm{B}$, AP-1, Sp1, whose binding sites are located within $2.2 \mathrm{kbp}$ upstream from transcription start site. We designed and synthesized two PIPs targeting MMP-9-NF- $\mathrm{B}$ binding site (-600 to -605) and MMP-9-AP-1 binding site (-70 to -77) (Fig. 3). In each experiment, we diluted them in dimethyl sulfoxide (DMSO) to make $10 \mathrm{mM}$ stock solutions.

Cell lines and culture conditions. The human renal cell carcinoma cell line Caki-2 was kindly transferred from Dr Cowell (Roswell Park Cancer Institute, NY, USA) and cultured in McCoy's 5A (Invitrogen Life Technologies, CA, USA) supplemented with 10\% FBS (McCoy's5A FBS PS), $100 \mathrm{U} / \mathrm{ml}$ penicillin, $100 \mu \mathrm{g} / \mathrm{ml}$ streptomycin and non-essential amino acids $(0.1 \mathrm{mM})$ under conditions of $5 \% \mathrm{CO}_{2}, 37^{\circ} \mathrm{C}$.

Real-time RT-PCR. Cells/well $\left(5.0 \times 10^{3}\right)$ of Caki-2 cells were plated in 6-well plates and cultured for $24 \mathrm{~h}$. Then PIPs were applied to the wells at the final concentration of 3 or $10 \mu \mathrm{M}$. No compound was applied to the negative control cells. After 48-h culture, the cells were washed with PBS and dissolved in TRIzol reagent (Invitrogen Life Technologies), then RNA was extracted following the manufacturer's instructions. After the treatment with DNase, first-strand cDNA was synthesized using iScript (Bio-Rad, Hercules, CA, USA) and real-time RT-PCR was carried out using SYBR Premix Ex Taq, Perfect Real-Time (Takara Bio, Otsu, Japan). The primers used in the real-time PCR to detect human MMP-9 were 5'-GAGACCGG TGAGCTGGATAG-3' (forward); 5'-TACACGCGAGTGAAG GTGAG-3' (reverse) and for human GAPDH were 5'-GCACC GTCAAGGCTGAGAAC-3' (forward); 5'-TGGTGAAGACGC CAGTGGA-3' (reverse). The relative quantity of the MMP-9 expression level was normalized by the expression level of GAPDH.

In vitro cell proliferation. Cells were seeded on 96-well microplates at the concentration of $3.0 \times 10^{3}$ cells per well and cultured at $37^{\circ} \mathrm{C}$ in $5 \% \mathrm{CO}_{2}$. Afterwards, cells were incubated for $72 \mathrm{~h}$ in the presence or absence of PIPs. Cell viability was evaluated by WST-8 (Nacalai Tesque, Japan) assay, in which absorbance at OD $450 \mathrm{~nm}$ was measured using with Wallac 1420 counter (Amersham Bioscience, Piscataway, NJ, USA).

Matrigel invasion assay. Invasion assay was carried out using BioCoat Matrigel invasion chambers (Becton-Dickinson Labware Co., MA, USA). Cells were suspended in culture medium at the concentration of $6.0 \times 10^{3}$ cells $/ \mathrm{ml}$ and $500 \mu \mathrm{l}$ of the suspension was seeded on the upper chamber. The lower chamber was filled with $500 \mu \mathrm{l}$ of the culture medium without cells. Cells were incubated for $48 \mathrm{~h}$ with or without PIPs. After removing non-invasive cells with a cotton swab, invasive cells adhering to membrane of the upper chamber were fixed and stained with Diff-Quick solution. Number of the cells on the membrane was counted under a light microscope at x 200 magnification. The counting was done for 10 fields per each membrane.

\section{MMP9-AP1 PI polyamide (HN50)}

\section{5'-CCCTGAGTCAGCACTTGCCT-3'

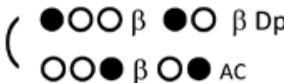

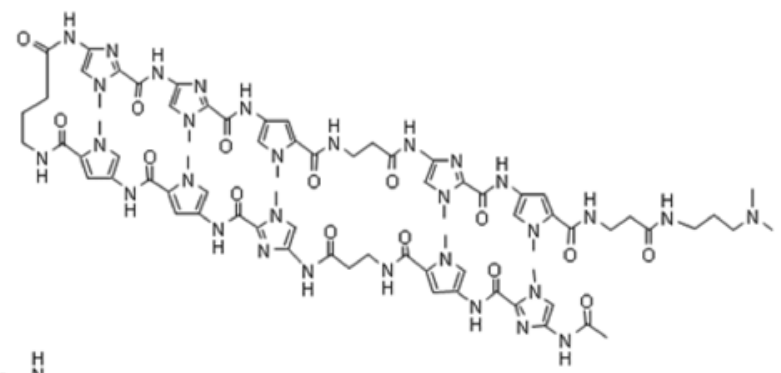

MMP9-NFkB PI polyamide (HN49)
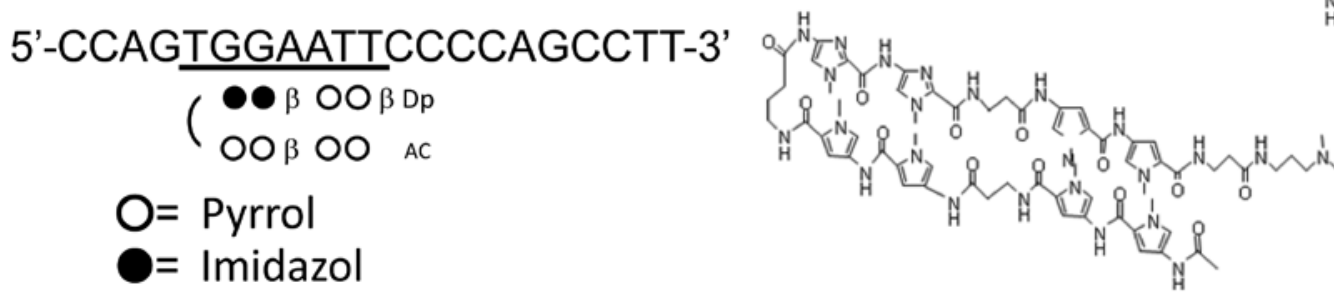

Figure 3. Structures of MMP-9-PIP targeting human MMP-9 promoter. MMP-9-AP-1 PIP and MMP-9-NF- $\kappa$ B PIP were designed to bind to the AP-1 binding site or NF- $\kappa$ B binding site, respectively, on human MMP-9 promoter. Target sequence and structure of both PIPs are shown. 


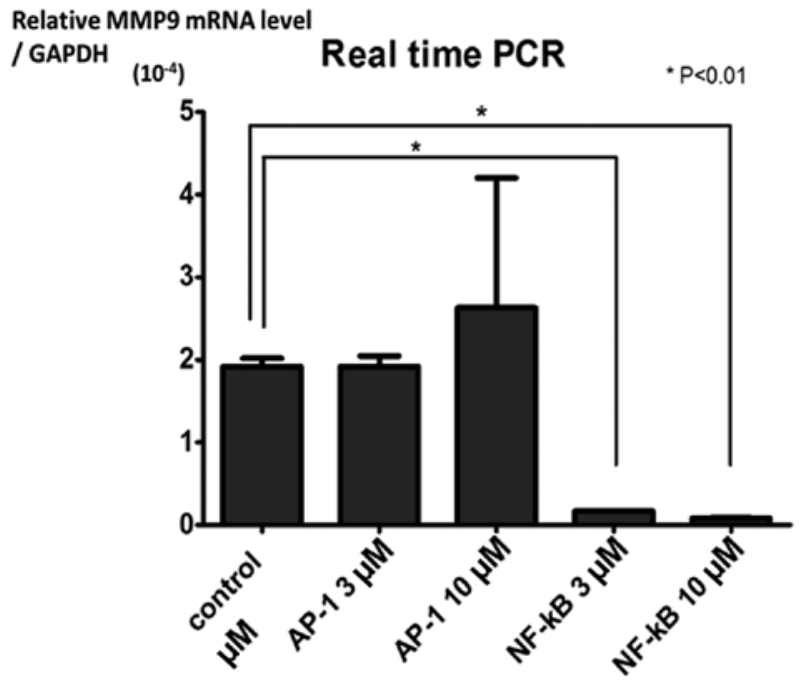

Figure 4. Expression level of MMP-9 in MMP-9-PIP treated cells. Caki-2 cells were treated with different concentration of MMP-9-PIPs for $48 \mathrm{~h}$, then, the expression level of MMP-9 was measured by real-time PCR. In comparison with the control group, expression of MMP-9 significantly decreased in the cells treated with 3 and $10 \mu \mathrm{M}$ of MMP-9-NF- $\mathrm{B}$ PIP $(\mathrm{P}<0.01)$. No significant change was observed in the cells treated with MMP-9-AP-1 PIP. Data are shown as the mean \pm SD of triplicate experiments.

Statistical analysis. All values are expressed as the mean \pm SE and the statistical significance was analyzed using the Student's t-test. A P-value of $<0.05$ was considered statistically significant.

\section{Results}

Immunohistochemistry. Two hundred and forty-nine of RCC specimens were subjected to immunohistochemical analysis to detect MMP-9 expression. One hundred and ninety-four out of 249 specimens $(78 \%)$ were classified to weak and 55 (22\%) to strong MMP-9 expression. Significant association was seen in a cancer-specific survival rate with the strength of the staining (Fig. 2). Patients in the strong stain group showed shorter survival than those in the weak group.

Expression of MMP-9 in MMP-9-PIP treated cells. We tested the expression level of MMP-9 in the cells treated with or without MMP-9 PIPs. The cells treated with 3 or $10 \mu \mathrm{M}$ of MMP-9-NF- $\mathrm{B}$ PIP showed significantly lower expression level than that of control cells $(\mathrm{P}<0.01)$ (Figs. 3 and 4). Even though the tendency of dose-dependency of MMP-9-NF- $\kappa \mathrm{B}$ PIP in the suppression of MMP-9 expression was observed, no significance was attained between 3 and $10 \mu \mathrm{M}$ (Fig. 4).

Cell proliferation in MMP-9-PIP treated cells. Cell viability was tested by WST8 assay $72 \mathrm{~h}$ after PIPs administration, however, no significant difference was found among the cells treated with MMP-9-NF- $\mathrm{B}$ PIP, MMP-9-AP-1 PIP and the control cells (Fig. 5). This result indicates that MMP-9 PIPs do not affect cell viability and proliferation activity.

Invasion assay for MMP-9-PIP treated cells. To evaluate the effect of PIPs on cell invasion activity, matrigel invasion assay was performed. The number of invaded cells in

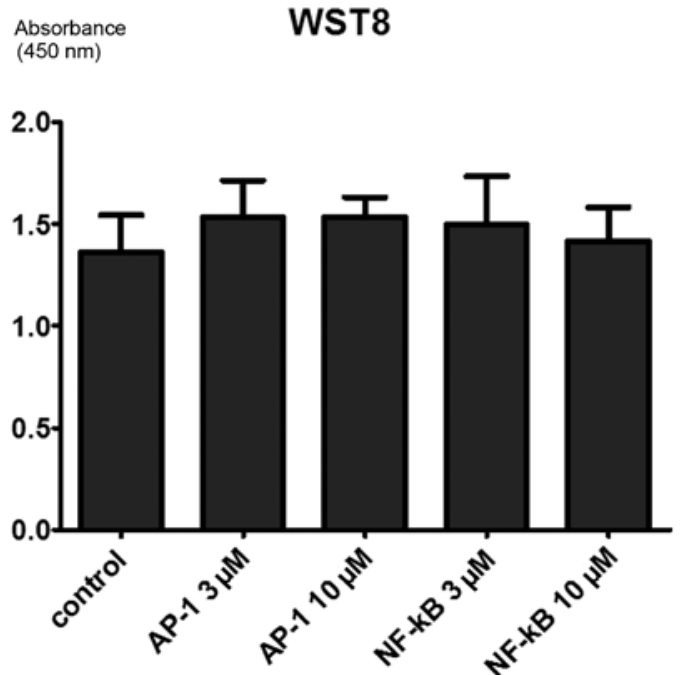

Figure 5. Viability of the cells treated with MMP-9-PIP. Caki-2 cells were treated with different concentration of MMP-9-PIPs for $72 \mathrm{~h}$ and the cell viability was measured by WST-8 assay. Neither MMP-9 AP-1 PIP nor MMP-9 NF- $\kappa$ B PIP treated cells showed significant difference in cell viability in comparison with the control cells. Data are shown as the mean \pm SD of triplicate experiments.

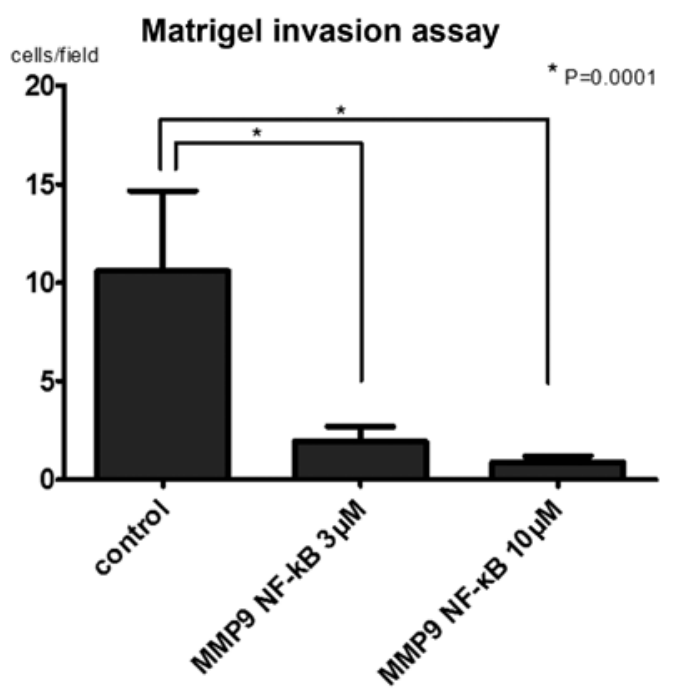

Figure 6. Invasion activity of the cells treated with MMP-9-PIPs. Caki-2 cells in Matrigel invasion chambers were treated with different concentration of MMP-9-PIPs for $48 \mathrm{~h}$ and the number of the cells invaded through the membranes was counted. Number of the invasive cells was significantly fewer in 3 and $10 \mu \mathrm{M}$ of MMP-9 NF- $\kappa \mathrm{B}$ PIP treated groups than in control. No significant difference was found between the cells treated with MMP-9 AP-1 PIP and non-treated cells. Data are shown as the mean \pm SD of triplicate experiments.

MMP-9-NF-кB PIP treated group were significantly lower than in control group $(\mathrm{P}<0.0001)$. Even though the tendency of dose dependency of MMP-9-NF-кB PIP in the suppression of MMP-9 expression was observed, there was no significant difference between 3 and $10 \mu \mathrm{M}$ (Fig. 6).

\section{Discussion}

In the present study, we confirmed an association of MMP-9 immunoreactivity and the cancer-specific survival rate by 
using clinical specimens. The patients with stronger immunoreactivity of MMP-9 showed shorter survival than patients with weaker immunoreactivity. The result suggests the importance of MMP-9 in survival of RCC patients. Moreover, Kawata et al suggested an association of MMP-9 expression between high nuclear grade (13) and systemic symptoms (14) in clinical cases of RCC. We also confirmed a similar tendency in this study (data not shown).

Some reports concerned an antitumor effect by reducing activity of MMP-9. They reduced MMP-9 expression using shRNA against co-regulator of the nuclear receptor for ovarian cancer proline-, glutamic acid-, leucine-rich protein-1 (PELP1) (20), or a monoclonal antibody against heat shock protein 90 (HSP90) of breast cancer cells (21). These reports confirmed an antitumor effect of MMP-9 in animal experiments. Our result and those reported encouraged us to develop PIPs targeting MMP-9.

We found suppressed expression of MMP-9 mRNA in Caki-2 cells after the PIP administration possibly by inhibiting transcription factor binding at the promoter domain. Though MMP-9-AP-1 PIP is reported to have suppressive effect in the cell lines of breast cancer, colon cancer and cervical cancer (19), only MMP-9-NF- $\mathrm{B}$ PIP showed suppression in the Caki-2 cell line. We presumed that mechanisms of the expression control vary among the cell types. Therefore, when designing PIP, detailed analysis of target gene transcription regulation is necessary to select an appropriate transcription factor for inhibition in each tumor and tumor type. Effective PIP may be decided from a candidate regulation domain of the targeting gene promoter.

For the evaluation of invasiveness of tumor in vitro, we performed Matrigel invasion assay, which has been commonly used to test cell invasion activity (22-24). We found that both 3 and $10 \mu \mathrm{M}$ of MMP-9-NF- $\kappa \mathrm{B}$ PIP restrained cell invasiveness significantly in comparison to control. These results were compatible with the result of the analysis of MMP-9 mRNA expression level, suggesting that MMP-9-NF- $\mathrm{B}$ PIP reduce the cell invasiveness by suppressing MMP-9 expression. Since Matrigel includes two of the major components of basement membrane, laminin and collagen IV, this result suggested the possibility to control cell invasion by administration of MMP-9-NF- $\kappa$ B PIP in the tissue.

The cell proliferation ability showed no significant difference between PIP treated group and control. The result does not contradict the fact that the main role of MMP-9 is degeneration of basement membrane and it is not directly participating in cell proliferation. However, Bauvois reported MMP-9 is not only involved in cell invasion but in cell proliferation and vascularization by an interaction of growth factor, cytokine and vascularization factor (25). Therefore, future studies are necessary including experiments concerning effects of MMP-9-PIP other than suppression of invasion.

We reported previously the in vitro and in vivo effect of MMP-9-PIP on breast cancer and colon cancer cell line (19). In the mouse model, intravenous injection of MMP-9 PIP clearly inhibited colon cancer metastasis to the liver. In that study, we also found that PIP remains in the cell nuclei for at least 6 days suggesting a continuous PIP effect. Antisense DNA or RNAi requires an adequate drug delivery system because it is easily disintegrated by nucleic acid degrading enzymes. However, PIP is consisted of N-methylpyrrole(Py) and N-methylimidazole (Im) and is uptaken by individual cells without special delivery system. It is stable without being a target of nucleic acid degrading enzymes. This should be a strong advantage for PIP.

Tyrosine kinase inhibitor (TKI) targeting mainly VEGF and the mTOR inhibitor are widely used for advanced renal cell carcinoma. However, most of them have adverse effects including skin reaction, hypertension, myeloablation, interstitial lung disease and sudden exacerbation at the time of withdrawal. Complete response is rarely seen and main effect of these molecular targeting agents at present is extension of progression-free survival and maintenance of the QOL of RCC patients. PIP may act supplementary for the effect of these molecular targeting agents and for reduction of its dosage because the mechanism of PIP is distinct from existing standard therapy (24). This is considered to be the second advantage.

In conclusion, MMP-9-NF- $\kappa$ B PIP in renal cell carcinoma cell line caki-2 suppressed its expression of MMP-9 and invasiveness. A possibility is suggested of MMP-9-NF- $\mathrm{B}$ PIP as a new therapeutic agent for renal cell carcinoma.

\section{Acknowledgements}

The authors thank Dr Yusuke Nagane and Dr Xiaofei Wang for their technical advice and valuable discussions.

\section{References}

1. Srinivasan R, Armstrong AJ, Dahut W, et al: Anti-angiogenic therapy in renal cell cancer. BJU Int 99: 1296-1300, 2007.

2. Board RE, Thistlethwaite FC and Hawkins RE: Anti-angiogenic therapy in the treatment of advanced renal cell cancer. Cancer Treat Rev 33: 1-8, 2007.

3. Figlin RA: Anti-angiogenic therapy in renal cell carcinoma: alone, in combination, or sequentially. Clin Adv Hematol Oncol 7: 662-665, 2009.

4. Kessenbrock K, Plaks V and Werb Z: Matrix metalloproteinases: regulators of the tumor microenvironment. Cell 141: 52-67, 2010.

5. Klein T and Bischoff R: Physiology and pathophysiology of matrix metalloproteases. Amino Acids 41: 271-290, 2011.

6. Bourboulia D and Stetler-Stevenson WG: Matrix metalloproteinases (MMPs) and tissue inhibitors of metalloproteinases (TIMPs): positive and negative regulators in tumor cell adhesion. Semin Cancer Biol 20: 161-168, 2010.

7. Lukaszewicz-Zajac M, Mroczko B and Szmitkowski M: Gastric cancer - the role of matrix metalloproteinases in tumor progression. Clin Chim Acta 412: 1725-1730, 2011.

8. Cho NH, Shim HS, Rha SY, et al: Increased expression of matrix metalloproteinase 9 correlates with poor prognostic variables in renal cell carcinoma. Eur Urol 44: 560-566, 2003.

9. Awakura Y, Ito N, Nakamura E, et al: Matrix metalloproteinase-9 polymorphisms and renal cell carcinoma in a Japanese population. Cancer Lett 241: 59-63, 2006.

10. Zhang X, Yamashita M, Uetsuki H, et al: Angiogenesis in renal cell carcinoma: evaluation of microvessel density, vascular endothelial growth factor and matrix metalloproteinases. Int J Urol 9: 509-514, 2002.

11. Sherief MH, Low SH, Miura M, et al: Matrix metalloproteinase activity in urine of patients with renal cell carcinoma leads to degradation of extracellular matrix proteins: possible use as a screening assay. J Urol 169: 1530-1534, 2003.

12. Kallakury BV, Karikehalli S, Haholu A, et al: Increased expression of matrix metalloproteinases 2 and 9 and tissue inhibitors of metalloproteinases 1 and 2 correlate with poor prognostic variables in renal cell carcinoma. Clin Cancer Res 7: 3113-3119, 2001. 
13. Kawata N, Nagane Y, Hirakata H, et al: Significant relationship of matrix metalloproteinase 9 with nuclear grade and prognostic impact of tissue inhibitor of metalloproteinase 2 for incidental clear cell renal cell carcinoma. Urology 69: 1049-1053, 2007.

14. Kawata N, Nagane $Y$, Igarashi $T$, et al: Strong significant correlation between MMP-9 and systemic symptoms in patients with localized renal cell carcinoma. Urology 68: 523-527, 2006.

15. Lai YM, Fukuda N, Ueno T, et al: Synthetic pyrrole-imidazole polyamide inhibits expression of the human transforming growth factor-beta1 gene. J Pharmacol Exp Ther 315: 571-575, 2005.

16. Kageyama Y, Sugiyama H, Ayame H, et al: Suppression of VEGF transcription in renal cell carcinoma cells by pyrrole-imidazole hairpin polyamides targeting the hypoxia responsive element. Acta Oncol 45: 317-324, 2006.

17. Matsuda H, Fukuda N, Ueno T, et al: Development of gene silencing pyrrole-imidazole polyamide targeting the TGF-beta1 promoter for treatment of progressive renal diseases. J Am Soc Nephrol 17: 422-432, 2006.

18. Minoshima M, Sasaki S, Fujimoto J, et al: Synthesis and biological properties of pyrrole-imidazole polyamide conjugates. Nucleic Acids Symp Ser (Oxf), pp35-36, 2007.

19. Wang X, Nagase H, Watanabe T, et al: Inhibition of MMP-9 transcription and suppression of tumor metastasis by pyrroleimidazole polyamide. Cancer Sci 101: 758-766, 2010.
20. Chakravarty D, Roy SS, Babu CR, et al: Therapeutic targeting of PELP1 prevents ovarian cancer growth and metastasis. Clin Cancer Res 17: 2250-2259, 2011.

21. Stellas D, El Hamidieh A and Patsavoudi E: Monoclonal antibody 4C5 prevents activation of MMP2 and MMP9 by disrupting their interaction with extracellular HSP90 and inhibits formation of metastatic breast cancer cell deposits. BMC Cell Biol 11: 51, 2010

22. Yan L, Kumagai SG, McGuire MH, et al: Protease activity and invasion of matrigel by the osteosarcoma-derived OSPR cell line. Biochem Soc Trans 22: S18, 1994.

23. Hall DMS and Brooks SA: In vitro invasion assay using matrigel(R). Methods Mol Med 58: 61-70, 2001.

24. Deryugina EI, Luo GX, Reisfeld RA, et al: Tumor cell invasion through matrigel is regulated by activated matrix metalloproteinase-2. Anticancer Res 17: 3201-3210, 1997.

25. Bauvois B: New facets of matrix metalloproteinases MMP-2 and MMP-9 as cell surface transducers: Outside-in signaling and relationship to tumor progression. Biochim Biophys Acta 1825: 29-36, 2011. 